\title{
WATTMETER METHODS OF MEASURING POWER EXPENDED UPON CONDENSERS AND CIRCUITS OF LOW POWER FACTOR.
}

By Edward B. Rosa.

The power factor of an alternating current flowing into and out of a good condenser, or a cable on open circuit, is so small as to make its measurement by a wattmeter somewhat difficult. Measurements made by the resonance method ${ }^{a}$ and by the calorimetric method ${ }^{b}$ gave power factors for some paraffined paper condensers of less than half of 1 per cent. These methods are, however, not adapted to general laboratory work. A simple wattmeter method of measuring power involves two corrections. First, for the power expended upon the fixed coil of the wattmeter, which is measured along with that of the condenser, and, second, for the change of phase of the potential current due to the combined inductance and capacity of the potential circuit. The correction (which is chiefly due to the inductance of the coil of the instrument which carries the potential current) is made small by using a large resistance in the potential circuit. Incandescent lamp filaments have extremely small inductance and capacity, and may be employed in series with one another, as the high resistance of the potential circuit; or wire coils may be employed if wound so as to avoid both inductance and capacity. The potential current, being very small, requires a relatively delicate suspension in order to give a satisfactory deflection. I bave found difficulty in securing sufficient stability and sensitiveness at once, and have hence been led to the use of a series of null methods for measuring the power factor of a condenser or cable current, thereby avoiding the measurement of a deflection, and dispensing with the requirement of so great stability. These methods were devised and employed in a long series of measurements in 1898, but the work was interrupted before its completion, and circumstances have since prevented me from resuming the work. 
The most obvious null method consists in using a variable inductance in the potential circuit. The difference of phase between the two currents being nearly $90^{\circ}$, if the potential current is slightly retarded by an added inductance the phase difference can be made $90^{\circ}$ and the deflection reduced to zero. Knowing the value of the added inductance, the frequency of the current, and the resistance, the change of phase, and hence the power factor, can be readily computed.

\section{USING AN AUXILIARY COIL ON THE WATTMETER.}

There are, however, several other methods of getting a difference of phase of $90^{\circ}$ and securing a zero deflection, any one of which can be employed for this purpose, according to the instruments one has available. These all depend upon the use of an auxiliary coil of fine wire wound over the fixed coil of coarse wire, having about the same number of turns as the fixed coil and made exactly equivalent to the fixed coil magnetically. This equivalence is shown by causing the same current to flow through the main coil and the auxiliary coil in opposite directions. The resulting magnetic field is then zero at the position of the suspended coil if the latter is not deflected when the current flowing through the main and auxiliary coils in opposite direction passes through the suspended coil also. Suppose, then, the condenser current $i_{1}$ passes through the fixed coil, the potential current $i_{2}$ passes through the suspended coil, and a small current $i_{3}$ (in phase with $i_{2}$ ) passes through the auxiliary coil. Then if $K$ is the constant of the instrument, the deflection $d_{1}$ of the suspended coil due to current $i_{1}$ in the main coil and $i_{2}$ in the suspended coil will be

$$
d_{1}=K i_{1} i_{2} \cos \phi_{1}
$$

where $\phi_{1}$ is the angular difference of phase between $i_{1}$ and $i_{2}$.

The current $i_{3}$ through the auxiliary coil would by itself (supposing there is no current in the main coil) produce a deflection $d_{2}$ such that

$$
d_{2}=K i_{2} i_{3} \text {. }
$$

If the current $i_{3}$ flows in such a direction as to make the deflection $d_{2}$ opposite to $d_{1}$, then when the currents $i_{1}$ and $i_{3}$ flow simultaneously, the deflection is the difference between $d_{1}$ and $d_{2}$; and when this is made zero by adjusting $i_{3}$ we have

$$
\begin{aligned}
i_{2} i_{3} & =i_{1} i_{2} \cos \phi_{1} \\
\therefore \quad \cos \phi_{1} & =\frac{i_{3}}{i_{1}}
\end{aligned}
$$


Another way of expressing this result is to say that the current $i_{3}$ (which is in phase with $i_{2}$, but reversed by the connectinns, and therefore differs in phase by $180^{\circ}$ from $i_{2}$ ) is added to $i_{1}$, and the vector sum is thereby made to differ by $90^{\circ}$ in phase from $i_{2}$. The ratio of $i_{3}$ to $i_{1}$ then gives the angle through which $i_{1}$ has been turned, to make it differ by $90^{\circ}$ from $i_{2}$. Or, again, the magnetic field of $i_{3}$, added to the magnetic field of $i_{1}$, gives a resultant magnetic field which differs by $90^{\circ}$ in phase from the potential current $i_{2}$. Hence, knowing $i_{3}$, we can find the power factor $\cos \phi_{1}$. The corrections for the resistance of the fixed coil and the inductance of the suspended coil being applied, we have $\cos \phi$, the true power factor.

The auxiliary or compensation current $i_{3}$ may be secured by several different devices, as follows:

(1) From the terminals of a noninductive resistance $r_{3}$ in the main circuit a shunt circuit is carried to the auxiliary coil, having a second condenser in series with it. Thus the current $i_{3}$ is proportional to the main current $i_{1}$, to the resistance $r_{3}$, to the capacity of the secondary condenser $C_{3}$, and to the frequency of the current. That is

$$
i_{3}=p C_{3} i_{1} r_{3} \text { and } \frac{i_{3}}{i_{1}}=\cos \phi_{1}=p C_{3} r_{3}
$$

Thus, knowing the capacity of the secondary condenser $C_{3}$, the frequency of the current $(p=2 \pi n)$, and the variable resistance $r_{3}$, we readily compute the power factor $\cos \phi_{1}$, which, corrected as before, gives $\cos \phi$.

(2) A portion of the potential current $i_{2}$ is shunted off through the auxiliary coil, and the value of $i_{3}$ becomes known if $i_{2}$ and the resistances of the divided circuit are known.

(3) By transforming down from the high e. m. f. $E$ impressed upon the condenser and shunt circuit, a small e. m. f. $e_{3}$ is obtained, differing in phase by $180^{\circ}$ almost exactly. A current $i_{3}=\frac{e_{3}}{R_{3}}$ is thus obtained, which differs in phase from the shunt current $i_{2}$ by $180^{\circ}$, and this is used as a compensation current. The resistance $R_{3}$ is varied until the deflection is zero, and $e_{3}$ is measured by an alternating current voltmeter; $i_{3}$ thus becomes known, and from it $\cos \phi_{1}$ as before.

When the capacity of the condenser is small, and therefore the main current $i_{1}$ is small, it may be better to pass $i_{1}$ through the suspended coil and the potential current through the fixed coil. This gives rise to two other arrangements of the circuits. But the principle is the same in every case, the compensation current $i_{3}$ being determined when the deflection of the wattmeter is zero, and the phase angle then found by a very simple calculation. 
The measurements which I made by these various methods on the same condenser's showed closely agreeing results, although the apparatus employed did not permit of measurements of the highest precision. I proceed to give a description of these methods, and a few examples of their use in measuring the power absorbed in some paraffined-paper condensers.

\section{METHOD 1.-SIMPLE DEFLECTION METHOD.}

The connections of the wattmeter to the source of alternating current and the condenser are shown in fig. 1 , and the phase diagram is shown in fig. 2. $F$ is the fixed coil and $M$ the movable coil of the wattmeter, $i_{1}$ the main condenser current, and $i_{2}$ the potential current

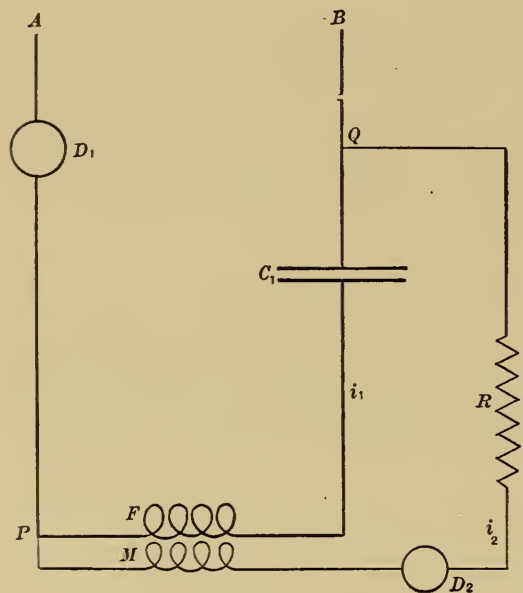

FIg. 1.-Connections for Method 1.

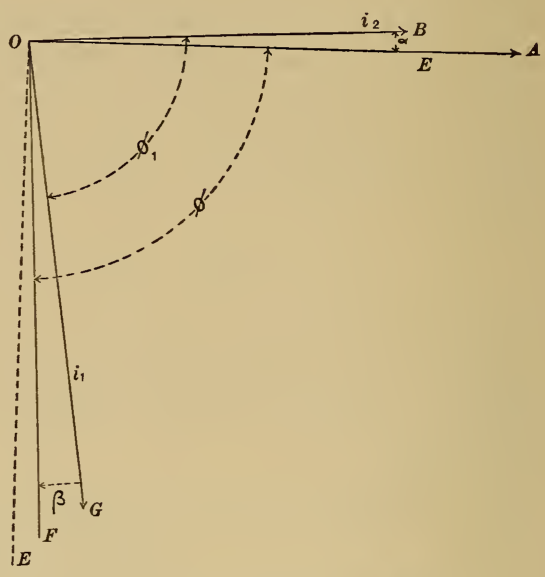

FiG. 2.-Phase diagram for Method 1.

passing through the large noninductive resistance $R$. An alternating potential of several hundred volts (up to 1,500 in my experiments) was applied to $A B . \quad D_{1}$ is the electrodynamometer or alternating current ammeter for measuring the current $i_{1}+i_{2}$, and $D_{2}$ measures $i_{2}$ alone. Inasmuch as these two currents differ in phase by nearly $90^{\circ}$, and $i_{2}$ is relatively small, the reading of $D_{1}$ is practically unaffected by. the current $i_{2}$. The noninductive resistance is several thousand ohms, and if it is known accurately, and also the voltage $E$, the instrument $D_{2}$ may be omitted and $i_{2}$ calculated from $E$ and $R$. The two coils $F$ and $M$ are joined together at the instrument in order that they may have the same potential and so avoid electrostatic attraction between them, a serious source of error when relatively high potentials are used, if the coils are not joined together. 
$O A$ (fig. 2) represents the phase of the electromotive force $E$. The potential current lags behind it by an angle $\alpha$, due to the inductance of the moving coil and of the resistance $R$. The high resistance itself often possesses a greater capacity than inductance, and hence the combined inductance of the circuit $\left(L_{2}\right)$ may be less than that of the moving coil of the wattmeter alone. It may even be negative, and therefore the potential current may be ahead in phase of $E$. In my experiments the inductance and capacity of the resistance $R$ were both very small, and $L_{z}$ was taken as that due to the known inductance of the moving coil of the wattmeter. The line $O G$ represents the condenser current $i_{1}$, differing in phase from $i_{2}$ by the angle $\phi_{1}$. $O F$ would have been the phase of this current if the fixed coil of the wattmeter had no resistance. The angle $\beta$ is the difference of phase $G O F$, due to this resistance, and $\tan \beta=r \div \frac{1}{p C}=p C r$, where $p=2 \pi n, n$ being the frequency of the alternating current, $C$ the capacity of the condenser, and $r$ the resistance of the wattmeter coil $F$ and the connections; that is, the resistance between $P$ and $Q$ when the condenser is short circuited. We then have the following expressions, $d$ being the deflection of the wattmeter, $K$ the constant of the latter, $\phi_{1}$ the measured difference of phase $B O G$ and $\phi$ the corrected difference $A O F$, due to the condenser.

$$
\begin{aligned}
& d=K i_{1} i_{2} \cos \phi_{1}, \text { or } \cos \phi_{1}=\frac{d}{K i_{1} i_{2}} \\
& \tan \alpha=\frac{p L_{2}}{R}, \tan \beta=p C r . \\
& \phi=\phi_{1}-\alpha+\beta
\end{aligned}
$$

As stated above, $\phi_{1}$ is the measured phase difference, and $\alpha$ and $\beta$ are respectively the small angular corrections due to the inductance of the potential circuit and the resistance of the fixed coils of the wattmeter

\begin{tabular}{|c|c|c|c|c|c|c|}
\hline $\begin{array}{l}\text { Condenser } \\
\text { employed. }\end{array}$ & $i_{1}$ & $i_{2}$ & $K$ & $d$ & $\cos \phi_{1}$ & $\begin{array}{c}\text { Power } \\
\text { factor, } \\
\text { uncorrected. }\end{array}$ \\
\hline Condenser No. 4.. & $\begin{array}{c}\text { Amperes. } \\
1.653\end{array}$ & $\begin{array}{c}\text { Amperes. } \\
0.1470\end{array}$ & 23300 & 26.0 & 0.00459 & $\begin{array}{l}\text { Per cént. } \\
0.459\end{array}$ \\
\hline Condenser No. $5 .$. & 3.494 & 0.1666 & 23100 & 76.0 & 0.00565 & 0.565 \\
\hline Condenser No. 6. & 3.494 & 0.1707 & 23100 & 75.0 & 0.00545 & $0.54 \check{ }$ \\
\hline
\end{tabular}

Table I.-Results by Method 1.

[ $n=120$ cycles; $E=1,400$ volts. $]$ 
and its connections. $\operatorname{Cos} \phi$ is then the power factor of the condenser. Table I gives some measurements made on three condensers, the second and third having a capacity about double the first.

The corrections $\alpha$ and $\beta$ are here very slight, so that $\phi$ is sensibly equal to $\phi_{1}$. The condensers are Stanley paraffined paper condensers, and No. $t$ has a sensibly smaller energy loss than the others.

\section{METHOD 2.-INDUCTANCE IN SHUNT CIRCUIT.}

Method 2 is similar to the first, except that a variable inductance $L_{3}$. fig. 3 , is inserted in the shunt circuit and the current $i_{2}$ is made to

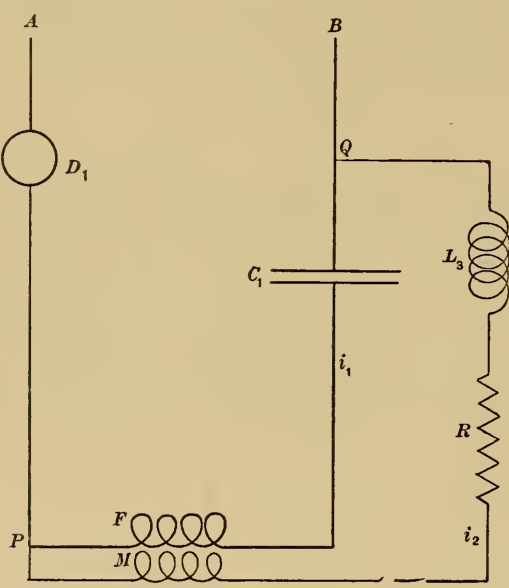

FIG. 3.-Connections for Method 2.

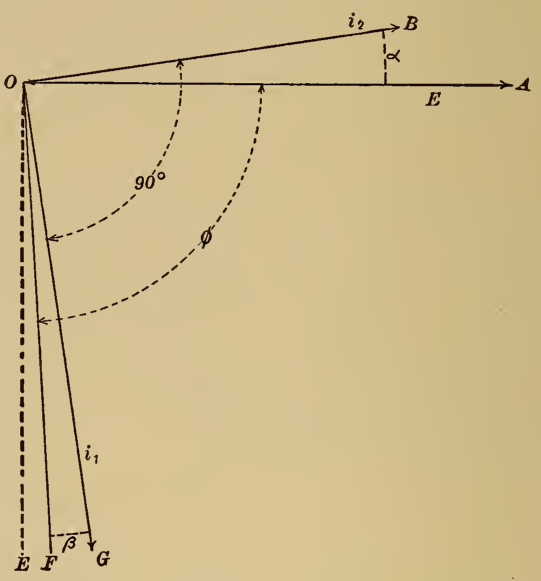

FIG. 4.-Phase diagram for Method 2.

lag by a larger angle than before, so that $\phi_{1}$ is now $90^{\circ}$, and the deflection is zero. If $L_{2}$ is the same as before and $L_{3}$ is the added inductance,

$$
\begin{aligned}
\tan \alpha & =\frac{p\left(L_{2}+L_{3}\right)}{R} \\
\tan \beta & =p C r \\
\phi & =90^{\circ}-\alpha+\beta
\end{aligned}
$$

This is a very convenient method if a variable known inductance of suitable magnitude is at hand, and it gives excellent results. The frequency of the current must be known more accurately than before, for $\alpha$ is now a much more important quantity than in method 1 , since the value of $\cos \phi_{1}$ depends mainly on $\alpha$. But $d, K, i_{1}$, and $i_{2}$, the quantities measured in method 1 , need not be measured at all in this method. 
Table II.-Results by Method 2.

[ $n=120, E=1,260$ volts. $]$

\begin{tabular}{|c|c|c|c|c|c|}
\hline Condenser employed. & $p=2 \pi n$ & $L_{2}+L_{3}$ & $R$ & $\cos \phi_{1}$ & $\begin{array}{c}\text { Power } \\
\text { factor, } \\
\text { uncorrected. }\end{array}$ \\
\hline Condenser No. 4 .. & 754 & $\begin{array}{l}\text { Henry. } \\
0.0530\end{array}$ & $\begin{array}{l}\text { Ohms. } \\
8,548\end{array}$ & 0.00468 & $\begin{array}{c}\text { Per cent. } \\
0.468\end{array}$ \\
\hline Condenser No. $5 \ldots$... & 754 & 0.0627 & 8,548 & 0.00553 & 0.553 \\
\hline
\end{tabular}

These results do not agree exactly with those by the first method. The difference is partly due to errors of the experiment, and partly to a change in the temperature of the condensers and of the wave form of the electromotive force employed. For want of a suitable variable inductance, very little was done with this method.

\section{METHOD 3. AUXILIARY CONDENSER SHUNT ON THE MAIN CIRCUIT.}

In this and the following methods the auxiliary compensation coil $\mathrm{A}$ is employed. As already explained, this coil is of fine wire and is

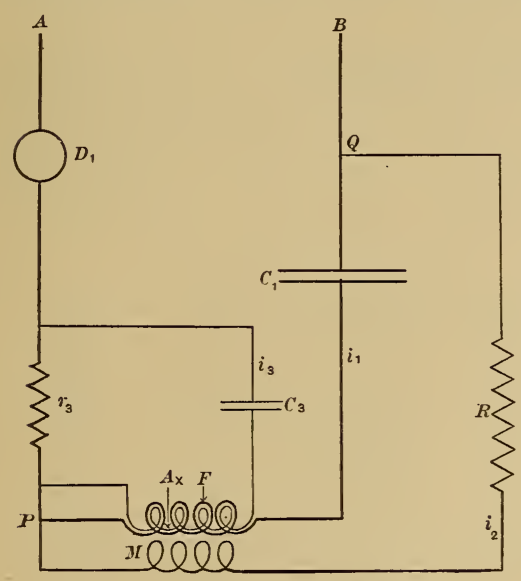

FIG. 5.-Connections for Method 3.

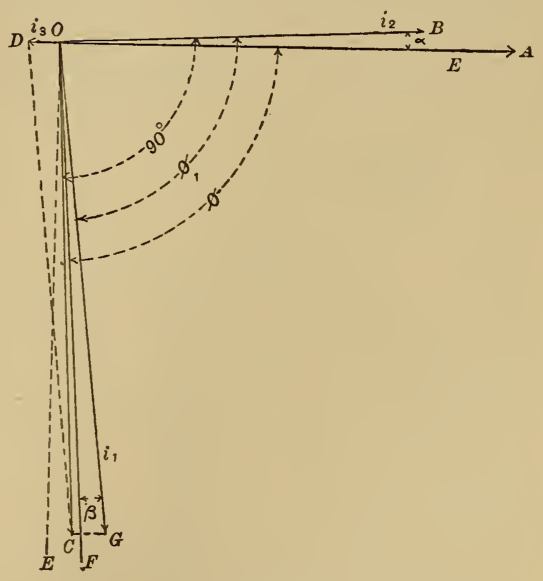

FIG. 6.-Phase diagram for Method 3.

wound on the outside of the main coil of the wattmeter, but thoroughly insulated from the latter. It is magnetically equivalent to the main coil, so that a current flowing in series through the two in opposite directions produces no magnetic field at the position of the moving coil.

A small variable resistance $r_{3}$, fig. 5 , is inserted in series with the main current $i_{1}$, and an auxiliary condenser $C_{3}$ is placed in series with the com- 
pensation coil on the terminals of this resistance. The compensation current $i_{3}$ is equal to $p C_{3} r_{3} i_{1}$, and is nearly $90^{\circ}$ in advance in phase of $i_{1}$; that brings it practically opposite in phase to $i_{2}$, as appears in fig. 6. We may compound $i_{1}$ and $i_{3}$ just as though they were in parallel in the same wire. The resultant is $O C$, and when $i_{3}$ has such a value that there is no deflection of the movable coil, $C O$ is exactly $90^{\circ}$ ahead of $O B$. Then, since $B O G=\phi_{1}$,

$$
\cos \phi_{1}=\frac{i_{3}}{i_{1}}=p C_{3} r_{3}
$$

Thus, it is unnecessary to measure $E, i_{1}, i_{2}$, or $R$, but only $C_{3}$ and $r_{3}$ in addition to the frequency of the current. To apply the corrections $\alpha$ and $\beta$ to obtain the value of $\phi$, it is of course necessary to determine $C, r$, and $R$ approximately, as in method 1 . The results obtained with five paraffined paper condensers are given in Table III. Nos. 2, 3, and 4 have a capacity of about 1.7 microfarads and 5 and 6 have a capacity of about 3.2 microfarads. These condensers were intended for 500 volt circuits. In these experiments, however, they were subjected to voltages between 910 and 1,386, most of the readings being taken at 1,260 . This is a very convenient method.

Table III.-Results by Method 3.

\begin{tabular}{|c|c|c|c|c|c|c|}
\hline $\begin{array}{l}\text { Condenser } \\
\text { employed. }\end{array}$ & $E$ & $i_{1}$ & $p=2 \pi n$ & $C_{3}$ & $r_{3}$ & $\cos \phi_{1}=p C_{3} r_{3}$ \\
\hline Condenser No. 2. & $\begin{array}{l}\text { Volts. } \\
1,260\end{array}$ & $\begin{array}{c}\text { Amperes. } \\
1.673\end{array}$ & 745 & $\begin{array}{c}\text { Farads. } \\
1.70 \times 10^{-6}\end{array}$ & $\begin{array}{c}\text { Ohms. } \\
3.46\end{array}$ & 0.00438 \\
\hline Condenser No. 2.... & 1,260 & 1. 686 & 745 & 1. $70 \times 10^{-6}$ & 3.48 & .00440 \\
\hline Condenser No. 3. & 1,260 & 1.633 & 745 & 1. $70 \times 10^{-6}$ & 3.48 & .00440 \\
\hline Condenser No. $3 . .$. & 1,260 & 1. 643 & 745 & 1. $70 \times 10^{-6}$ & 3.48 & .00440 \\
\hline Condenser No. 4 . . & 1,386 & 1. 756 & 745 & 1. $70 \times 10^{-6}$ & 3.50 & .00443 \\
\hline Condenser No. 4.... & 1,260 & 1. 673 & 745 & 1. $70 \times 10^{-6}$ & วิ. 52 & .00445 \\
\hline Condenser No. $2+3$ & 1,260 & 3. 305 & 745 & 1. $70 \times 10^{-6}$ & 3. 62 & .00458 \\
\hline Condenser No. $3+4$ & 1,260 & 3.286 & 745 & 1. $70 \times 10^{-6}$ & 3.66 & .00463 \\
\hline Condenser No. 5... & 910 & 2.329 & 745 & 1. $70 \times 10^{-6}$ & 4.35 & .00551 \\
\hline Condenser No. $5 . .$. . & 910 & 2. 319 & 745 & $1.70 \times 10^{-6}$ & 4.36 & .00552 \\
\hline Condenser No. $5 . .$. . & 1,260 & 3.176 & 745 & 1. $70 \times 10^{-6}$ & 4.26 & .00540 \\
\hline Condenser No. 6.... & 910 & 2.277 & 745 & 1. $70 \times 10^{-6}$ & 4.20 & .00531 \\
\hline Condenser No. 6.... & 1,260 & 3.146 & 745 & 1. $70 \times 10^{-6}$ & 4.12 & .00521 \\
\hline Condenser No. $5+6$. & 910 & 4.810 & 745 & 1. $70 \times 10^{-6}$ & 4.50 & .00570 \\
\hline
\end{tabular}




\section{EFFECT OF THE SELF AND MUTUAL INDUCTANCE OF THE FIXED AND AUXILIARY COILS.}

The self-inductance of the fixed coils of the wattmeter employed in these measurements is 0.075 millihenry, of the auxiliary coil 0.111 millihenry, and the mutual inductance of the two is 0.072 millihenry. At a frequency of $120, p=754$, and the reactance of the auxiliary coil is $0.083 \mathrm{ohm}$. This is wholly negligible in its effect upon the magnitude or phase of the compensation current $i_{3}$. The self-inductance of the fixed coil has no appreciable effect upon the magnitude or phase of the main current $i_{1}$. The mutual inductance between the coils, however, may have an appreciable effect upon the magnitude of the compensation current $i_{3}$ in the auxiliary coil, provided the electromotive force $e_{3}$ on the compensation circuit is small. Thus, the back e. m. f. in this circuit, due to the current $i_{1}$ in the fixed coil, is $p M i_{1}$, and this is equal to 0.16 volt when $i_{1}=3$ amperes and $p=754$. In methods 4 and $5, e_{3}$ was usually 45 to 70 volts, and hence the correction $\gamma$ due to mutual inductance is only one or two units in the last decimal place of the values given for $\cos \phi_{1}$ in Tables IV and V. In method 3 , however, $e_{3}$ was smaller and the correction is 5 to 6 in the last decimal place of the values of $\cos \phi_{1}$ in Table III.

The expression for $\cos \phi_{1}$ in which the mutual inductance of the fixed and auxiliary coils is taken into account, is

$$
\begin{aligned}
& \text { For Method 3, } \cos \phi_{1}=\frac{i_{3}}{i_{1}}=p C\left(r_{3}-p M\right) \\
& \text { For Method 4, } \cos \phi_{1}=\frac{i_{3}}{i_{1}}=\frac{\left(\frac{i_{1}}{i_{2}} r_{3}-p M\right)}{R_{3}+r_{3}} \\
& \text { For Method 5, cos } \phi_{1}=\frac{i_{3}}{i_{1}}=\frac{\left(\frac{e_{3}}{i_{1}}-p M\right)}{R_{3}}
\end{aligned}
$$

There is also a slight correction $\delta$ to be made in method 3 , for the potential current $i_{2}$, which also passes through $r_{3}$; that is, the resultant of $i_{1}$ and $i_{2}$ passes through $r_{3}$. This makes a slightly larger current and shifts the phase a little. The shifting of the phase, however, does no appreciable harm. The correction $\gamma$ can be made insignificant by increasing $r_{3}$ and using an auxiliary condenser of correspondingly smaller capacity, while the correction $\delta$ is reduced to an insignificant quantity by having the potential current $i_{2}$ sufficiently small in comparison with $i_{1}$. In fact, it is easy under most circumstances to eliminate the three corrections $\beta, \gamma, \delta$ by properly proportioning the coils, 
leaving only a small correction $\alpha$ to be applied, due to the resistance of the fixed coils and connections.

METHOD 4.-SHUNT ON POTENTIAL CIRCUIT.

In this case, fig. 7 , the compensation current $i_{3}$ is shunted off from $i_{2}$, the resistance $r_{3}$ being in the potential circuit through the moving

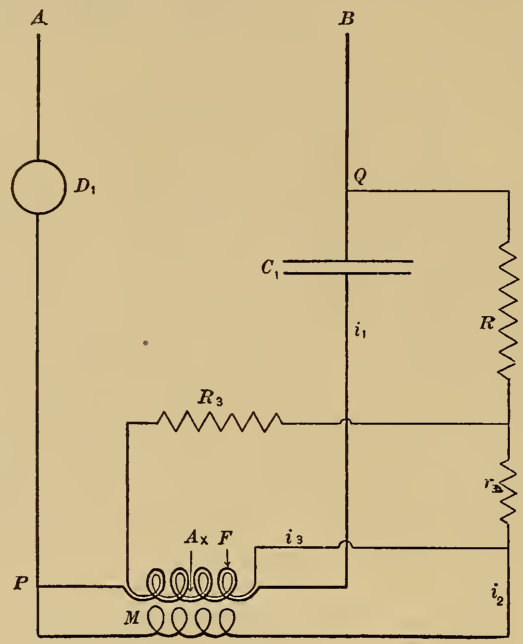

Fig. 7.-Connections for Method 4.

coil instead of the main circuit as in method 3. The compensation current is thus in phase with $i_{2}$, but by reversing the terminals of the compensation coil it has the same effect as though it were opposite in phase. Thus, fig. 6 represents this method also.

Therefore, since

$$
i_{3}=i_{2} \frac{r_{3}}{R_{3}+r_{3}}, \cos \phi_{1}=\frac{i_{3}}{i_{1}}=\frac{i_{2}}{i_{1}} \frac{r_{3}}{R_{3}+r_{3}}
$$

Table IV.-Results by Method 4.

$[\mathrm{E}=1,260$ volts. $]$

\begin{tabular}{|c|c|c|c|c|c|c|}
\hline Condenser employed. & $i_{1}$ & $i_{2}$ & $r_{3}$ & $R_{3}$ & $\cos \phi_{1}$ & $\begin{array}{l}\text { Power } \\
\text { factor } \\
\text { uncor- } \\
\text { rected. }\end{array}$ \\
\hline Condenser No. 5 & $\begin{array}{c}\text { Amperes. } \\
3.016\end{array}$ & $\begin{array}{c}\text { Ampere. } \\
0.1396\end{array}$ & $\begin{array}{c}\text { Ohms. } \\
507\end{array}$ & 3800 & 0.00544 & $\begin{array}{c}\text { Per cent. } \\
0.544\end{array}$ \\
\hline Condenser No. 5 ..... & 3. 037 & 0.1729 & 507 & 4830 & 0.00540 & 0.540 \\
\hline Condenser No. 6 ..... & 2.962 & 0.1735 & 507 & 5100 & 0.00529 & 0.529 \\
\hline
\end{tabular}


METHOD 5. USING A TRANSFORMER FOR THE COMPPENSATION CURRENT.

In this case the high potential winding of a transformer is joined across the terminals $A B$ of the circuit, fig. 8, and th 3 low potential

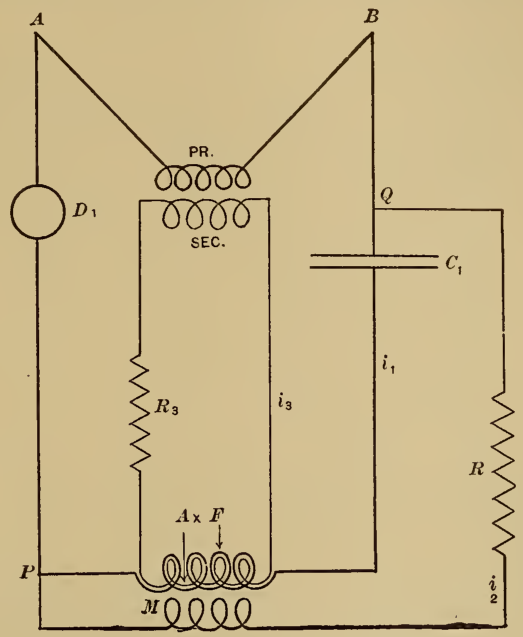

FIG 8.-Connections for Method 5.

coil supplies the current $i_{3}$ to the compensation coil through a resistance $R_{3}$. This current is opposite in phase to $i_{2}$, as in methods 3 and 4.

$$
\begin{aligned}
i_{3} & =\frac{e_{3}}{R_{3}} \\
\cos \phi_{1} & =\frac{i_{3}}{i_{1}}=\frac{e_{3}}{i_{1} R_{3}}
\end{aligned}
$$

The small electromotive force $e_{3}$ may be determined by the ratio of transformation, or by direct measurement. It is very convenient to put a voltmeter on the secondary of the transformer and measure $e_{3}$ directly, and then get $E$ by multiplying by the ratio of transformation. Some of the results obtained by this method are given in Table V.

The transformer used here is the potential transformer which was employed to get the voltage on the main condenser circuit. In these experiments it had a ratio of about 14, and hence, for 90 volts on the secondary there was 1,260 on the primary. The secondary consisted of two equal coils, and the compensation coil was joined to the terminals of one of them. The slight current used (about a hundredth of an ampere) did not alter the ratio of transformation of the transformer. 
METHOD 6. SHUNT ON MAIN CIRCUIT.

This method corresponds to method 4 , but the condenser is placed in the moving coil circuit, fig. 9 , and a portion of $i_{1}$ is shunted off into

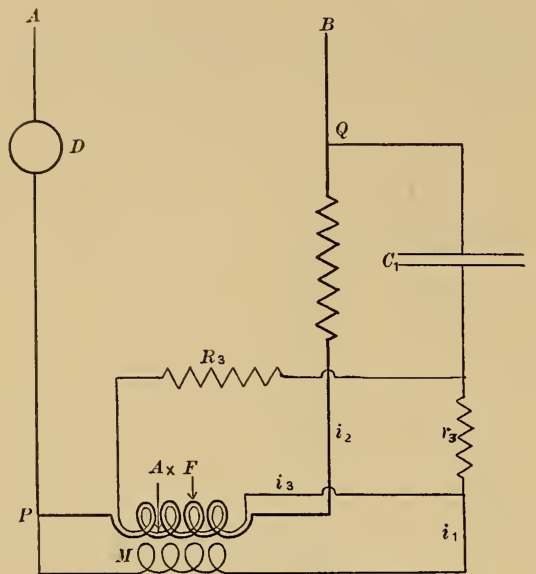

Fig. 9.-Connections for Method 6.

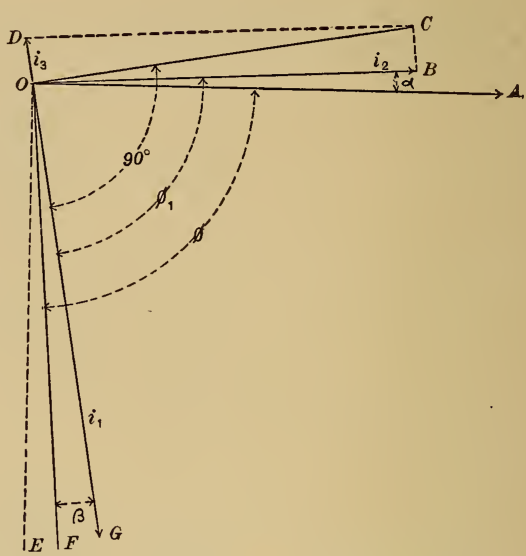

FIG. 10.-Phase diagram for Method 6.

the compensation coil. Thus, $i_{3}$ is opposite in phase to $i_{1}$ and is combined with $i_{2}$ so that the resultant of $i_{2}$ and $i_{3}, O C$, is $90^{\circ}$ different in phase from $i_{1}$, fig. 10 .

Thus:

$$
i_{3}=i_{1} \frac{r_{3}}{R_{3}+r_{3}}
$$

This method is especially adapted to small condensers, where the current $i_{1}$, is very small and $i_{2}$ can be increased above its usual value.

Table V.-Results by Method V. .

[ $E=1,260$ volts $=$ voltage on the condensers. $e=45$ volts $=$ voltage on the compensation circuit. $]$

\begin{tabular}{|c|c|c|c|c|}
\hline Condensers employed. & $R_{3}$ & $i_{3}=\frac{e}{R_{3}}$ & $i_{1}$ & $\cos \phi_{1}=\frac{i_{3}}{i_{1}}$ \\
\hline 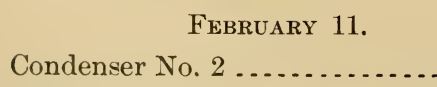 & 6000 & $\begin{array}{l}\text { Ampere. } \\
0.00750\end{array}$ & $\begin{array}{c}\text { Amperes. } \\
1.633\end{array}$ & 0.00459 \\
\hline Condenser No. 3 . . . . . . . . & 6250 & 0.00720 & 1. 614 & 0.00446 \\
\hline Condenser No. 4 ..... & 6000 & 0.00750 & 1.633 & 0.00459 \\
\hline Condenser No. $2+3 \ldots$ & 2950 & 0.01525 & 3. 206 & 0.00476 \\
\hline Condenser No. $3+4 \ldots$. & 2965 & 0.01517 & 3.181 & 0.00477 \\
\hline Condenser No. 5 . . . ..... & 2560 & 0.01758 & 3.146 & 0.00559 \\
\hline Condenser No. 6 . . . . . . . & 2730 & 0.01648 & 3.089 & 0.00533 \\
\hline Condenser No. $5+6 \ldots$. & 1310 & 0.03435 & 6.330 & 0.00542 \\
\hline
\end{tabular}


Table V.-Results by Method V-Continued.

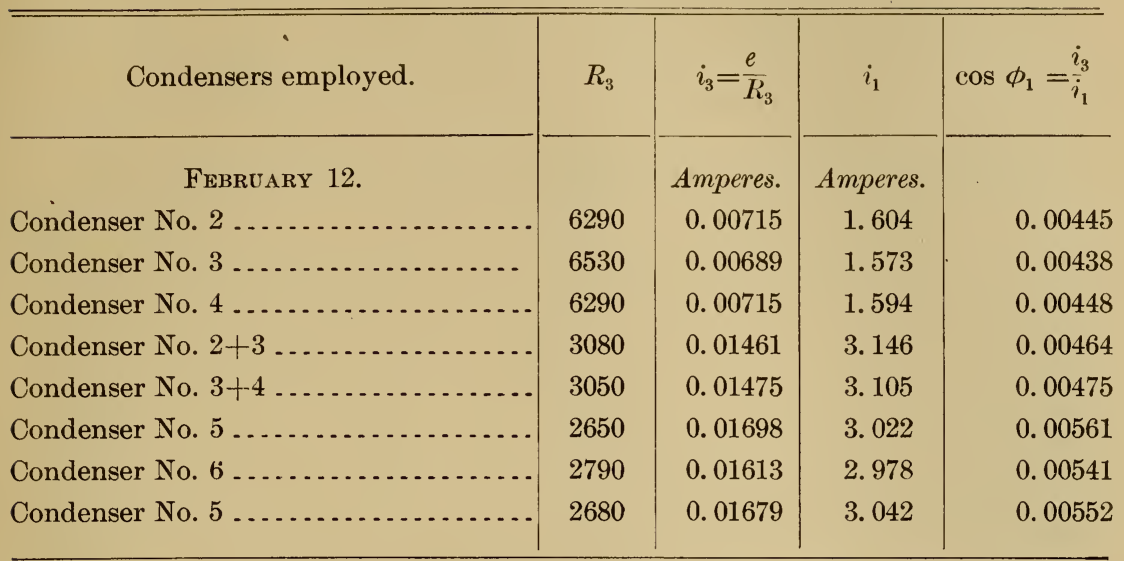

METHOD 7. AUXILIARY CONDENSER ON TRANSFORMER.

This corresponds to method 5 , where the transformer is used as a source of $i_{3}$, but as in method 6 , the condenser under test is placed in the moving coil circuit. In order to bring the compensation current $90^{\circ}$ out of phase with $i_{2}$, with which it is combined, an auxiliary con-

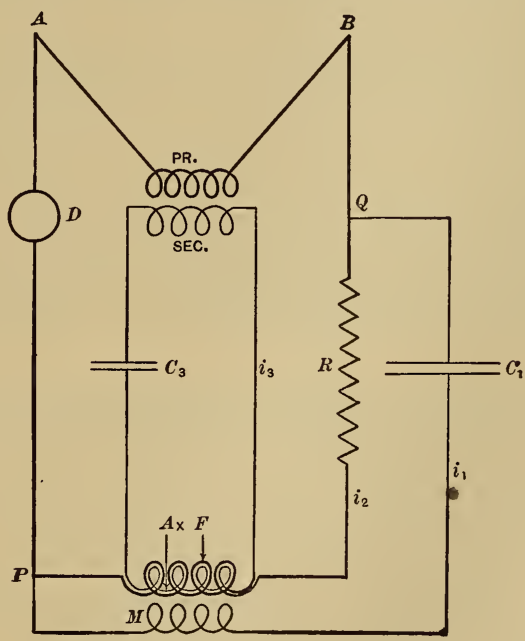

Fig. 11.-Connections for method 7.

denser $C_{3}$ is placed in the $i_{3}$ circuit instead of a resistance, fig. 11 . The phases are shown in fig. 10 . Thus:

$$
\begin{array}{lc}
i_{3}=p C_{3} e_{3} & i_{2}=\frac{E}{R} \\
\cos \phi_{1}=\frac{i_{3}}{i_{2}}=p C_{3} e_{3} \frac{R}{E}=\frac{p C_{3} R}{n}
\end{array}
$$


where $n$ is the ratio of transformation of the potential transformer.

Or, the formula may be written $\cos \phi_{1}=\frac{p C_{3} e_{3}}{i_{2}}$, and $e_{3}$ determined directly by a voltmeter and $i_{2}$ by an ammeter or electrodynamometer.

Table VI.-Sumuary of Quantities to be Measured in the Varjous METHODS.

\begin{tabular}{|c|c|c|c|c|c|c|c|c|c|c|c|}
\hline Method 1. & $d$ & $K$ & $i_{1}$ & $i_{2}$ & & & & & & & \\
\hline Method 2 & $\cdots$ & & $\cdots$ & & $p$ & $I$ & $R$ & & & & \\
\hline Nethod 3 & & & 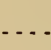 & $\cdots$ & $p$ & & & $r_{3}$ & $C_{3}$ & & \\
\hline Method 4 & $\cdots$ & & $i_{1}$ & $i_{2}$ & $\ldots$ & & & $r_{3}$ & & $R_{3}$ & \\
\hline Method 5 & & & $i_{1}$ & $\cdots$ & 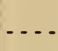 & & & 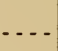 & & $R_{3}$ & $e_{3}$ \\
\hline Method 6 & & & $i_{1}$ & $i_{2}$ & .. & & & $r_{3}$ & ….. & $R_{3}$ & \\
\hline Method 7 & & & & $i_{2}$ & $p$ & & & $\cdots$ & $C_{3}$ & $\cdots \cdot$ & $e_{3}$ \\
\hline
\end{tabular}

The quantities in Table VI are as follows:

$d$ is the deflection of the electrodynamometer in scale divisions.

$K$ is the constant of the electrodynamometer.

$i_{1}$ is the main current, through the condenser under test.

$i_{2}$ is the potential current, through the large resistance $R$.

$p$ is $2 \pi$ times the frequency.

$L$ is the variable inductance added to the potential circuit.

$R$ is the large potential resistance, as free as possible from inductance and capacity.

$r_{3}$ is the small resistance shunted by the auxiliary circuit.

$C_{3}$ is the auxiliary condenser, of constant value.

$R_{3}$ is the resistance in series with the auxiliary coil.

$e_{3}$ is the relatively small electromotive force supplying the auxiliary current.

Thus it appears that each method requires the determination of three or four quantities, and a choice of method will be determined in part by what instrumental facilities are available for the work. All the methods are capable of giving good results, but the null methods are more satisfactory than method 1 , unless one has a wattmeter which is both sensitive and stable, and which has a nearly uniform field, so that the constant $K$ does not vary too rapidly. All the methods require the two correction terms $\alpha$ and $\beta$ to be applied to derive the true power factor $\cos \phi$ from the measured power factor $\cos \phi_{1}$. These two corrections are of opposite sign, as already explained, and should be small. In the experiments here described the resistance of the fixed coils of the wattmeter and the leads through which the main 
current flowed - that is, from $P$ to $Q$ with the condenser short circuited (figs. 1, 3, 5, etc.) -was $0.08 \mathrm{ohm}$. When the current is 1.6 amperes, $i^{2}{ }^{r}{ }^{r}$ is 0.20 watt, and this requires a correction of about 0.0001 in the power factor. The correction for inductance in the moving coil is of the same order of magnitude and hence the results of the measurements cited above as examples are not very different from the true power factor $\cos \phi$. At the time these measurements were made I did not have facilities for measuring the various quantities involved in these several methods with sufficient accuracy to make a crucial test of the methods. The results, however, show that the various methods agree substantially, and there is no reason to doubt the entire reliability of any of them. The values given above for the power factor are somewhat larger than those found for the same condensers by the calorimetric method, ${ }^{a}$ but in those experiments the high electromotive force impressed upon the condensers was obtained by resonance from a lower electro-motive force and the harmonics were therefore largely suppressed. In all the work described in this article the high electro-motive force was obtained by transforming up, and hence the harmonics were retained and magnified by the condensers. Since the power factor is higher for higher frequencies, it is higher for the harmonics and therefore for the distorted wave. This is one of the subjects to be investigated when this work is resumed.

By taking careful note of the temperature of the condensers, and determining accurately the frequency of the current and the exact values of the $\alpha$ and $\beta$ corrections, we would obtain not only a crucial test of the various methods, but also data as to how the energy losses vary with the frequency, temperature, and wave form. I hope soon to repeat these measurements with improved apparatus, and hope to obtain results of sufficient precision to give valuable information of this character. 


